\title{
FDG-PET/CT lymph node staging after neoadjuvant chemotherapy in patients with adenocarcinoma of the esophageal-gastric junction
}

\author{
Pavel Fencl, ${ }^{1}$ Otakar Belohlavek, ${ }^{1}$ Tomas Harustiak, ${ }^{2}$ Milada Zemanova ${ }^{3}$ \\ ${ }^{1}$ PET Center, Hospital Na Homolce, Roentgenova 2, 15030 Prague 5, Czech Republic \\ ${ }^{2} 3$ rd Clinic of Surgery, First Faculty of Medicine, Charles University in Prague and University Hospital Motol, V Uvalu 84, Praha 5 \\ 150 06, Czech Republic \\ ${ }^{3}$ Clinic of Oncology, First Faculty of Medicine, Charles University in Prague and General Teaching Hospital, U Nemocnice 2, \\ Praha 2128 08, Czech Republic
}

\begin{abstract}
Objectives: The aim of the analysis was to assess the accuracy of various FDG-PET/CT parameters in staging lymph nodes after neoadjuvant chemotherapy.

Methods: In this prospective study, 74 patients with adenocarcinoma of the esophageal-gastric junction were examined by FDG-PET/CT in the course of their neoadjuvant chemotherapy given before surgical treatment. Data from the final FDG-PET/CT examinations were compared with the histology from the surgical specimens (gold standard). The accuracy was calculated for four FDG-PET/CT parameters: (1) hypermetabolic nodes, (2) large nodes, (3) large-and-medium large nodes, and (4) hypermetabolic or large nodes.

Results: In 74 patients, a total of 1540 lymph nodes were obtained by surgery, and these were grouped into 287 regions according to topographic origin. Five hundred and two nodes were imaged by FDG-PET/CT and were grouped into these same regions for comparison. In the analysis, (1) hypermetabolic nodes, (2) large nodes, (3) large-andmedium large nodes, and (4) hypermetabolic or large nodes identified metastases in particular regions with sensitivities of $11.6 \%, 2.9 \%, 21.7 \%$, and $13.0 \%$, respectively; specificity was $98.6 \%, 94.5 \%, 74.8 \%$, and $93.6 \%$, respectively. The best accuracy of $77.7 \%$ reached the parameter of hypermetabolic nodes. Accuracy decreased to $62.0 \%$ when also smaller nodes (medium-large) were taken for the parameter of metastases. Conclusions: FDG-PET/CT proved low sensitivity and high specificity. Low sensitivity was based on low
\end{abstract}

Correspondence to: Pavel Fencl; email: pavel.fencl@homolka.cz detection rate $(32.6 \%)$ when compared nodes imaged by FDG-PET/CT to nodes found by surgery, and in inability to detect micrometastases. Sensitivity increased when also medium-large LNs were taken for positive, but specificity and accuracy decreased.

Key words: FDG-PET/CT-Esophageal

adenocarcinoma-Nodal

staging - Accuracy—Neoadjuvant chemotherapy

\author{
Abbreviations \\ R0 \\ Radical resection of the cancer \\ FDG 18F-fluoro-deoxy-glucose \\ SCC Squamous cell carcinoma \\ AC Adenocarcinoma (of the esophagus) \\ PERCIST 1.0 PET response criteria in solid \\ tumors, version 1.0 \\ EUS Endoscopic ultrasonography
}

There is little doubt that the malignant involvement of lymph nodes is a key factor in the outcome of patients with esophageal carcinoma following radical (R0) resection [1-4]. However, some disagreement remains as to the optimal method for nodal staging in esophageal cancer according to the AJCC scheme [5-7]. Endoscopic ultrasonography (EUS) is currently considered a more sensitive tool than computed tomography [7-9] or contrast-enhanced CT [5] for imaging lymph nodes. 
Metabolic information from integrated FDG-PET/CT may improve the sensitivity of anatomic evaluation by CT $[10,11]$, and some authors have reported good results in imaging lymph node metastases using FDG [12-14], but others have not been able to show that such data are helpful $[9,15]$. A number of studies have also been performed in patients with squamous cell carcinoma of the esophagus [16] (SCC) or with "esophageal cancer" [17]. These patients have various ratios of SCC and adenocarcinoma (AC) of the esophagus, and as these types of carcinoma differ substantially $[2,18,19]$, the results of these studies are only partially applicable to patients with AC. Confronting our FDG-PET/CT data with histologic specimens, we concluded FDG-PET/CT to be of low sensitivity and of high specificity and that is why we tried to verify our opinion using a large cohort of patients using region-based analysis.

\section{Materials and methods}

\section{Patient participants}

One hundred and fifty-three patients with adenocarcinoma of the esophageal-gastric junction were prospectively recruited to the study between January 2009 and February 2013 to ascertain the metabolic response to neoadjuvant chemotherapy. Initial FDG-PET/CT staging was followed by FDG-PET/CT restaging after the first course of therapy and no earlier than 14 days after the third course of neoadjuvant chemotherapy. The data from postchemotherapy examinations were used in the analysis. Seventy-four patients (6 female, 68 male; median age 60 years; range $27-74$ years) were surgically resected and were selected for this analysis. The participants were all of middle European origin; of similar socioeconomic background; and without deficits in education, living standard, access to health care, or medical information.

\section{Therapy}

Patients received three preoperative and three postoperative cycles of chemotherapy, with surgery performed 36 weeks after completing the third cycle. Intravenous chemotherapy consisted of epirubicin $\left(50 \mathrm{mg} / \mathrm{m}^{2}\right)$ and cisplatin $\left(60 \mathrm{mg} / \mathrm{m}^{2}\right)$ with hydration and standard antiemetic prophylaxis on day 1 plus continuous infusion of fluorouracil (200 mg/m ${ }^{2}$ daily) for 21 days (ECF) using a portable infusion pump or oral capecitabine $(1.000 \mathrm{mg} /$ $\mathrm{m}^{2}$ twice daily) for 14 days (ECCap) in a 21 days cycle (according to the MAGIC regimen) [20, 21]. Depending on the localization and extent of the tumor, all patients underwent either transthoracic esophagectomy with gastric pull-up reconstruction and intrathoracic esophagogastric anastomosis (Ivor-Lewis procedure), or prox- imally extended total or proximal gastrectomy with transhiatal resection of the distal esophagus and suprahiatal anastomosis. All patients that underwent resection had negative proximal resection margins as determined by intraoperative frozen histopathological analysis. Abdominal D1 plus lymphadenectomy (all lymph node tissue around the left gastric artery, common hepatic artery, celiac trunk, and proximal half of the lienal artery) was a standard part of all operations; radical infracarinal mediastinal lymphadenectomy was added to transthoracic esophagectomy.

\section{Lymph node imaging}

Patients fasted for at least $6 \mathrm{~h}$ before intravenous administration of FDG. Blood glucose levels were determined in each patient before FDG administration. The dose of FDG was corrected for patient weight as recommended by the European Association of Nuclear Medicine [22]. Examinations were performed without the application of iodinated contrast material intravenously. Patients were positioned in a supine head-first position on the table of a Biograph 40 TruePointTrueV HD PET/ CT scanner (Siemens, Erlangen, Germany). For CT data acquisition, the following parameters were used: $120 \mathrm{kV}$, $63 \mathrm{mAs}$ (effective), collimation $24 \times 1.2 \mathrm{~mm}$, and slice thickness $5 \mathrm{~mm}$. Axial images were reconstructed in $2 \mathrm{~mm}$ increments using both B19S and B50F kernels. In all examinations, $3 \mathrm{~min} /$ bed position, Gaussian filtration, kernel $5 \mathrm{~mm}$, matrix size $168 \times 168$ were used for PET data acquisition. Using the Siemens TrueX (PSF) reconstruction algorithm, PET data were reconstructed in corrected and noncorrected images, with $2 \mathrm{~mm}$ reconstruction increments, three iterations, and 21 subsets. The PERCIST 1.0 protocol was used to maintain reproducibility as described previously $[23,24]$.

\section{Surgical sampling of lymph nodes}

In 52 out of 74 patients $(70.3 \%)$ the carcinoma was classified as Siewert type I, in 17 out of 74 patients (23.0\%) as Siewert type II, and in 5 out of 74 patients (6.7\%) as Siewert type III [25]. Altogether, 1540 lymph nodes were surgically resected and subjected to histological analysis. According to the origin of the specimens, denoted by operating surgeons, topographical regions were identified. Following the principles of lymph node classification [26], lymph nodes were assorted into these five regions: region 1, subcarinal nodes; region 2, periesophageal and preaortal nodes of the lower mediastinum; region 3, left and right pericardial nodes; region 4, perigastric nodes along the lesser curvature and nodes around the left gastric artery; region 5, perivascular nodes. In total, 287 regions (mean 3.8 regions per patient) comprised the matrix used in the analyses [27]. 
Lymph nodes in every region were counted and histologically categorized as metastatic or nonmetastatic.

\section{Lymph node diagnosis by FDG-PET/CT}

In the design of the prospective study according to the PERCIST 1.0 protocol [23], no more than two of the metastatic lymph nodes were analyzed in each patient. To enable comparison between nodes imaged after the chemotherapy and those surgically removed, examinations were re-evaluated from digitally archived data. The short axis of identified nodes was measured, and FDG consumption was compared with the background. In this way, all nodes were categorized according to the diameter of the short axis either large $(>1 \mathrm{~cm})$, small $(<0.5 \mathrm{~cm})$, or medium-large $(0.5-1 \mathrm{~cm})$, or, according to their metabolic activity, hypermetabolic (FDG consumption that exceeded the consumption in surrounding tissue) [28] or nonhypermetabolic. Next, the nodes were sorted into a matrix identical to that of the surgical specimens. All evaluations were performed by a single radiologist with more than 8 years experience in PET/CT and 20 years in $\mathrm{CT}$ diagnostic imaging.

\section{Comparison between the nodes identified by $F D G-P E T / C T$ and the ones surgically resected}

Histological evaluation (gold standard) was considered positive when at least one metastatic lymph node was found in a region. FDG-PET/CT evaluation was considered positive when at least one lymph node bore pertinent parameters in the analyzed region. Four FDGPET/CT parameters were compared to the gold standard: (1) hypermetabolic nodes, (2) large nodes, (3) largeand-medium large nodes, and (4) hypermetabolic or large nodes. Any small and nonhypermetabolic lymph node was considered negative. For statistical analyses, regions where lymph nodes were not imaged by FDG$\mathrm{PET} / \mathrm{CT}$ were also classified as negative.

\section{Results}

\section{Accuracy in the detection of lymph nodes metastases}

Only 502 of 1540 surgically resected lymph nodes $(32.6 \%)$ were also imaged by FDG-PET/CT (Table 1). Comparative data for the four FDG-PET/CT parameters and the gold standard have been tabulated (Table 2). Three of the tested parameters gave similar accuracy: hypermetabolic nodes (77.7\%), hypermetabolic/or large nodes $(74.8 \%)$, and large nodes $(72.5 \%)$. Despite the fact that the parameter large-and-medium large nodes gave the best sensitivity (21.7\%), it proved the worst specificity (74.8\%) and accuracy (62.0\%), which therefore made it the least reliable for detection of metastases. The other three parameters produced very few false positives with the specificity range from $93.6 \%$ to $98.6 \%$.

In the analysis, hypermetabolism proved better for detecting metastatic regions than node size. The single metabolic parameter, hypermetabolic nodes, proved positive predictive value of $72.7 \%$, whereas the single anatomic parameter, large nodes, proved positive predictive value of $14.3 \%$. Neither combination of parameters "medium-and-large LNs" nor "hypermetabolic or large nodes" reached the level of metabolic single parameter when showed positive predictive value of $21.4 \%$ and of $39.1 \%$, respectively.

\section{False-negative and false-positive findings}

False negatives were primarily due to the inability of FDG-PET/CT to image all lymph nodes found at surgery. Less frequent was the situation when nodes were imaged but not recognized as metastatic due to low metabolic activity or diameter shorter than $1.0 \mathrm{~cm}$ [9]. Only once, large lymph nodes adjacent to the tumor mass were differentiated from the tumor itself in region 4.

Four out of 218 nonmetastatic regions (1.8\%) were falsely identified as metastatic ones. In three of them, different types of inflammation were found: a granulomatous inflammation with anthracosis in region 1, necrotic LNs with foamy histiocytes along with microscopic ulceration of the tumor in region 4, and histiocytosis in region 5. No histologic explanation could be found for one region [29].

\section{Discussion}

The exclusive selection of patients does not allow a general conclusion for all esophageal cancers to be drawn; on the other hand, our data were not subject to variability due to biologically different cancers in different localizations.

We were confronted with a low number of lymph nodes differentiated in CT scans. Although this observation is not new in the literature $[8,18]$, the count of identified nodes was below our expectation. FDG-PET/ CT examinations were carried out at high quality, carefully checked for high degree of standardization [24] according to PERCIST 1.0 protocol and fulfilled current EANM recommendations [22]. We did not find a way to improve FDG-PET/CT scans technically by changing the parameter settings.

The use of more than one radiologist would increase the reliability of the analysis from the statistical point of view. With a respect to the anticipated low sensitivity and high specificity, we dismissed it as a redundant intent. Even if a substantially better reader had made the double-reading, the increased sensitivity would have been from clinical point of view still unacceptably low and 
Table 1. Differences in lymph nodes surgically resected and imaged by FDG-PET/CT, divided by regions

\begin{tabular}{lllllll}
\hline & Region 1 & Region 2 & Region 3 & Region4 & Region 5 \\
\hline N. resected & 293 & 270 & 215 & 334 & 428 & 1540 \\
N. imaged & 146 & 91 & 90 & 90 & 85 & 502 \\
$(\%)$ & 49.8 & 33.7 & 41.9 & 26.9 & 19.9 & 32.6 \\
\hline
\end{tabular}

N. resected: lymph nodes (LNs) surgically resected, N. imgaged: LNs imaged by FDG-PET/CT, Region 1: subcarinal LNs, Region 2: periesophageal LNs of lower mediastinum, Region 3: paracardial LNs, Region 4: perigastric LN, Region 5: perivascular LNs

Table 2. Sensitivity, specificity, and accuracy of all four tested parameters

\begin{tabular}{|c|c|c|c|c|c|c|}
\hline & $\mathrm{GS}+$ & GS- & Total & & Estimated value & CI95\% \\
\hline \multicolumn{7}{|c|}{ (1) Hypermetabolic LNs } \\
\hline & & & & Sensitivity & 11.6 & $5.49-22.1$ \\
\hline Test + & 8 & 4 & 12 & Specificity & 98.6 & $95.7-99.6$ \\
\hline Test- & 61 & 214 & 275 & Accuracy & 77.7 & $72.3-82.3$ \\
\hline \multirow[t]{2}{*}{ Total } & 69 & 218 & 287 & PPV & 72.7 & $39.3-92.7$ \\
\hline & & & & NPV & 77.9 & $72.4-92.7$ \\
\hline \multicolumn{7}{|c|}{ (2) Large LNs } \\
\hline & & & & Sensitivity & 2.9 & $0.5-11.0$ \\
\hline Test + & 2 & 12 & 14 & Specificity & 94.5 & $90.35-97.0$ \\
\hline Test- & 67 & 206 & 273 & Accuracy & 72.5 & $66.8-77.5$ \\
\hline \multirow[t]{2}{*}{ Total } & 69 & 218 & 287 & PPV & 14.3 & $2.5-43.8$ \\
\hline & & & & NPV & 75.5 & $69.8-80.3$ \\
\hline \multicolumn{7}{|c|}{ (3) Medium-and-large LNs } \\
\hline & & & & Sensitivity & 21.7 & $13.1-33.6$ \\
\hline Test + & 15 & 55 & 70 & Specificity & 74.8 & $68.4-80.3$ \\
\hline Test- & 54 & 163 & 217 & Accuracy & 62.0 & $56.1-67.6$ \\
\hline \multirow[t]{2}{*}{ Total } & 69 & 218 & 287 & PPV & 21.4 & $12.9-33.2$ \\
\hline & & & & NPV & 75.1 & $68.7-80.6$ \\
\hline \multicolumn{7}{|c|}{ (4) Hypermetabolic/large LNs } \\
\hline & & & & Sensitivity & 13.0 & $6.5-23.8$ \\
\hline Test + & 9 & 14 & 23 & Specificity & 93.6 & $89.2-96.3$ \\
\hline Test- & 60 & 204 & 264 & Accuracy & 74.2 & $68.7-79.1$ \\
\hline \multirow[t]{2}{*}{ Total } & 69 & 218 & 287 & PPV & 39.1 & $20.5-61.2$ \\
\hline & & & & NPV & 77.3 & $71.6-82.1$ \\
\hline
\end{tabular}

$G S$ gold standard: GS + regions with metastatic lymph nodes (LNs) found by histology; GS - regions without metastatic LNs; test + region where at least one LN bears tested parameter; test- negative test; $S E$ sensitivity, $P P V$ positive predictive value, $N P V$ negative predictive value; CI 95\%: confidence interval valid for $95 \%$ of data

specificity would have remained still excellent. On the other hand, when a substantially worse reader had made the same, specificity would have dropped slightly, but it would have been probably still very good, and sensitivity would have remained unacceptably low.

A cogent reason for the use of high-resolution imaging (i.e., endoscopic ultrasonography) in nodal staging is the fact that more small lymph nodes can be imaged in the vicinity of the probe. Such a method could improve the ratio of imaged/removed lymph nodes and increase sensitivity $[30,31]$. That is why we tested whether increased sensitivity based on imaging of smaller objects would be also able to improve accuracy in the search for metastases. We set the threshold for the presence of metastases down when medium-large nodes $0.5-1.0 \mathrm{~cm}$ were also taken for positive. Using the threshold sensitivity increased 7.5 times compared to the single anatomic parameter. But, an increase of sensitivity was accompanied by a decrease in specificity of $20 \%$ and accuracy of $15 \%$.
The next weak point in the diagnostics was the inability of FDG uptake imaging to prove metastases in imaged, but nonhypermetabolic nodes [28]. In our study, the parameter of hypermetabolism proved higher accuracy than node size. Unfortunately, the metabolic information was not sensitive enough in these diagnostics, but on the other hand was trustworthy due to the small number of false-positive findings. PET quantification using SUV is of special importance when measuring the effect of the treatment. We have used the PERCIST 1.0 in a longitudinal follow-up of lesions in the prospective study. In this paper, we have concentrated on a single-time point after chemotherapy and before surgery. In a single-time point scenario, utilization of SUV is of a limited value, when assessing relatively small lymph nodes, where the partial volume effect artificially lowers the response of the scanner. In this situation, a subjective assessment is more appropriate in deciding whether the uptake has increased above that of the adjacent soft tissue. Therefore we decided for this type of assessment. 

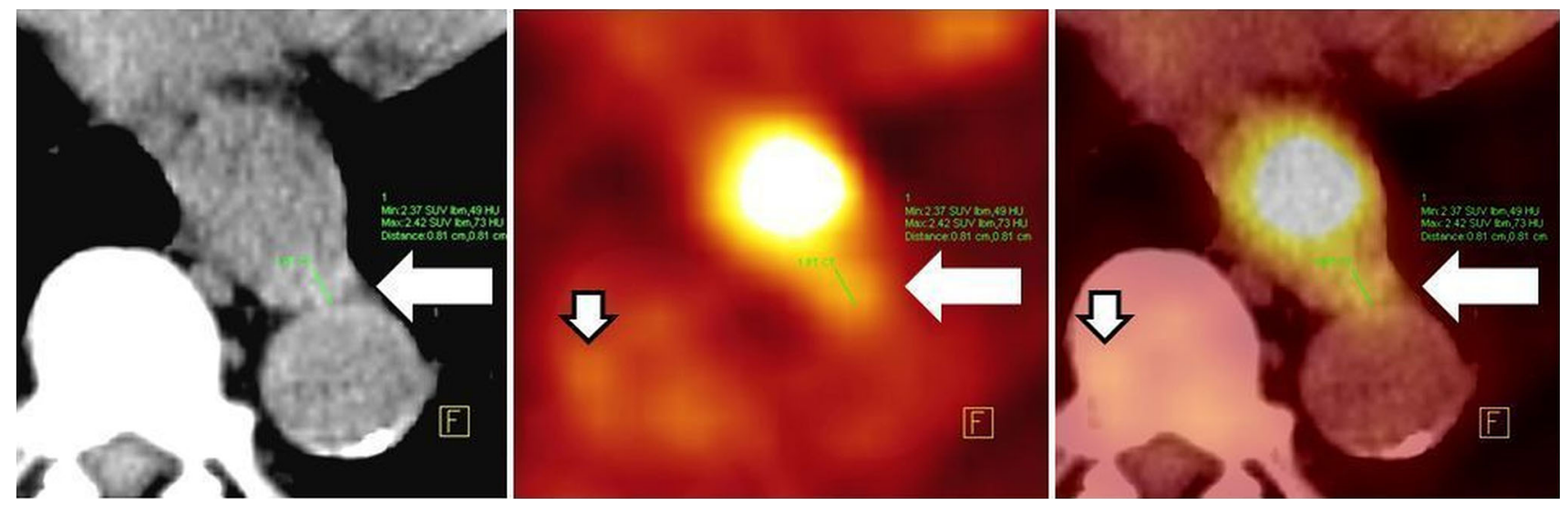

Fig. 1. FDG-PET/CT imaging of small hypermetabolic lymph node. A (CT picture): small node with the short axis of $0.8 \mathrm{~cm}$ (arrow); B (FDG-PET picture): small hypermetabolic spot near to esophageal cancer, three similar spots are in the

FDG-PET/CT is a hybrid diagnostic tool where metabolic information provided by PET enhances the anatomic one provided by $\mathrm{CT}$ and vice versa. Without hybrid imaging, the reader would not be able to localize small hypermetabolic spots to the lymph node $[14,32]$ and differentiate small LNs as metastatic ones (Fig. 1). The simultaneous ("hybrid") reading of both modalities is a robust tool in a nodal staging in routine practice, despite its low sensitivity.

As a result of our examinations, surgeons looked pointedly for lymph nodes with excessive metabolism, where metastases were present at $98.6 \%$. Unfortunately it only occurred in $11.6 \%$ of regions. In other regions, surgeons sampled nodes blindly according to the standard protocol.

The low detection rate of removed and imaged LNs suggested the imaging with higher resolution should be used for lymph nodes identification. But the only anatomic improvement of imaging methods will probably be devalued by an increase of false positivity. The data analyzed in the study indicated that to reach higher accuracy, a higher resolution in lymph nodes imaging should be accompanied by an improvement of other nonanatomic parameters whether metabolic (new radiopharmaceuticals), molecular (MR spectroscopy, DWI), or others. The identification of smaller lymph nodes alone does not improve the accuracy.

\section{Conclusion}

In a homogenous group of 74 patients with adenocarcinoma of the esophageal-gastric junction, nodal staging by FDG-PET/CT was not accurate due to the inability of FDG-PET/CT to image all lymph nodes and to verify micrometastases or metastases in small lymph nodes. The parameter "hypermetabolic nodes" proved to be of low sensitivity, but high specificity due to the low number vicinity (small arrow); C (FDG-PET/CT picture): fused image diagnosed metastatic lymph node (arrow), and physiologic glucose metabolism in the bone marrow.

of false-positive findings. Inflammation in lymph nodes mimicking metastases played a marginal role in this group of patients. The parameters "large nodes", "largeand-medium large nodes", and "large or hypermetabolic nodes" did not improve accuracy for low sensitivity, low specificity, or both. Due to low sensitivity, negative FDG-PET/CT findings could not exclude the presence of metastases; high specificity indicates that any positive nodal staging must be carefully considered.

Acknowledgments. The authors Pavel Fencl, Tomas Harustiak, and Milada Zemanova received the support of the grant of Ministry of Health of the Czech Republic. NT12331-5/2011. The authors thank Mr. Ray Swann for correcting the English version of the manuscript. Open access was supported by Ministry of Health, Czech Republic - conceptual development of research organization (Nemocnice Na Homolce - NNH, 00023884), PVI7501IPMZ

\section{Compliance with ethical standards}

Conflict of interest The author declares that they have no competing interests.

Ethical approval The study was approved by ethics committees from all three participating hospitals, and all patients provided signed informed consent forms before inclusion and before any FDG-PET/CT examinations.

Open Access This article is distributed under the terms of the Creative Commons Attribution 4.0 International License (http://creativeco mmons.org/licenses/by/4.0/), which permits unrestricted use, distribution, and reproduction in any medium, provided you give appropriate credit to the original author(s) and the source, provide a link to the Creative Commons license, and indicate if changes were made.

\section{References}

1. Yuequan J, Shifeng C, Bing Z (2010) Prognostic factors and family history for survival of esophageal squamous cell carcinoma patients after surgery. Ann Thorac Surg 90:908-913 
2. Dubecz A, Kern M, Solymosi N, Schweigert M, Stein HJ (2015) Predictors of lymph node metastasis in surgically resected T1 esophageal cancer. Ann Thorac Surg 99:1879-1886

3. Gillies RS, Middleton MR, Han C, Marshall RE, et al. (2012) Role of positron emission tomography-computed tomography in predicting survival after neoadjuvant chemotherapy and surgery for oesophageal adenocarcinoma. Br J Surg 99:239-245

4. Xu XL, Zheng WH, Zhu SM, Zhao A, Mao WM (2015) The prognostic impact of lymph node involvement in large scale operable nodepositiveesophageal squamous cell carcinoma patients: a 10-year experience. PLoS ONE 15:10. doi:10.1371/journal.pone.0133 076.eCollection

5. Sobin L, Gospodarowicz M, Wittekind Ch (eds) (2009) International Union Against Cancer (UICC) TNM classification of malignant tumours, 7th Edn. New York: Wiley, pp 66-72

6. Ngamruengphong S, Sharma VK, Nguyen B, Das A (2010) Assessment of response to neoadjuvant therapy in esophageal cancer: an updated systematic review of diagnostic accuracy of endoscopic ultrasonography and fluordeoxyglucose positron emission tomography. Dis Esophagus 23:216-231

7. van Vliet EP, Heijenbrok-Kal MH, Hunink MG, Kuipers EJ, Siersema PD (2008) Staging investigations for esophageal cancer: a meta-analysis. Br J Cancer 98:547-557

8. Walker AJ, Spier BJ, Perlman SB, et al. (2011) Integrated PET/CT fusion imaging and endoscopic ultrasound in the pre-operative staging and evaluation oesophageal cancer. Mol Imaging Biol 13:166-171

9. Cuellar SL, Carter BW, Macapinlac HA, et al. (2014) Clinical paging of patients with early esophageal adenocarcinoma: does FDG-PET/CT have a role? J Thorac Oncol 9:1202-1206

10. Okada M, Murakami T, Kumano S, et al. (2009) Integrated FDG$\mathrm{PET} / \mathrm{CT}$ compared with intravenous contrast-enhanced CT for evaluation of metastatic regional lymph nodes in patients with resectable early stage esophageal cancer. Ann Nucl Med 23:73-80

11. Cordin J, Lehmann K, Schneider PM (2010) Clinical staging of adenocarcinoma of the esophago-gastric junction. Recent Results Cancer Res 182:73-83

12. Cerfolio RJ, Bryant AS, Ohja B, Bartolucci AA, Eloubeidi MA (2005) The accuracy of endoscopic ultrasonography with fine-needle aspiration, integrated positron emission tomography with computed tomography, and computed tomography in restaging patients with esophageal cancer after neoadjuvant chemoradiotherapy. J Thorac Cardiovasc Surg 129:1232-1241

13. Hsu PK, Link KH, Wang SJ, et al. (2011) Preoperative positron emission tomography/computed tomography predicts advanced lymph node metastasis in esophageal squamous cell carcinoma patients. World J Surg 35:1321-1326

14. Shin S, Kim HK, Choi YS, Kim K, Shim YM (2014) Clinical stage T1-T2N0M0 oesophageal cancer: accuracy of clinical staging and predictive factors for lymph node metastasis. Eur J Cardiothorac Surg 46:274-279

15. Shimizu S, Hosokawa M, Itoh K, et al. (2009) Can hybrid FDG$\mathrm{PET} / \mathrm{CT}$ detect subclinical lymph node metastasis of esophageal cancer appropriately and contribute to radiation treatment planning? A comparison of image-based and pathological findings. Int $\mathbf{J}$ Clin Oncol 14:421-425
16. Wei Ch, Deng W, Li N, et al. (2015) Lymph node ratio as an alternative to the number of metastatic lymph nodes for the prediction of esophageal carcinoma patient survival. Dig Dis Sci . doi: 10.1007/s10620-015-3681-1

17. Thurau K, Palmes D, Franzius C, et al. (2011) Impact of PET-CT on primary staging and response control on multimodal treatment of esophageal cancer. World J Surg 35:608-616

18. Gillies RS, Middleton MR, Maynard ND, Bradley KM, Gleeson FV (2011) Additional benefit of ${ }^{18} \mathrm{~F}$-fluorodeoxyglucose integrated positron emission tomography/computed tomography in the staging of esophageal cancer. Eur Radiol 21:274-280

19. Cho JW, Choi SC, Jang JY, et al. (2014) Lymph node metastases in esophageal carcinoma: an endoscopist's view. Clin Endosc 47:523-529

20. Cunningham D, Allum WH, Stenning SP, et al. (2006) Perioperative chemotherapy versus surgery alone for resectable gastresophageal cancer. N Engl J Med 355:11-20

21. Pechačová Z, Zemanová M, Haruštiak T, Vítek P, Fencl P (2013) Peri-operative chemotherapy in patients with esophageal and gastro-esophageal junction cancer-three years of experience. Prague Med Rep 114:57-71

22. Boellaard R, O'Doherty MJ, Weber WA, et al. (2010) FDG PET and PET/CT: EANM procedure guidelines for tumour PET imaging: version 1.0. Eur J Nucl Med Mol Imaging 37:181-200

23. Wahl RL, Jacene H, Lodge MA (2009) From RECIST to PERCIST: evolving considerations for PET response criteria in solid tumors. J Nucl Med 50:122S-150S

24. Fencl P, Belohlavek O, Harustiak T, Zemanova M (2012) The analysis of factors affecting the threshold on repeated 18F-FDG$\mathrm{PET} / \mathrm{CT}$ investigations measured by the PERCIST protocol in patients with esophageal carcinoma. Nucl Med Commun 33:1188-1194

25. Siewert JR, Feith M, Werner M, Stein HJ (2000) Adenocarcinoma of the esophagogastric junction. Results of surgical therapy based on anatomical/topographic classification in 1,002 consecutive patients. Ann Surg 232:353-361

26. Hofstetter W, Correa AM, Bekele N, et al. (2007) Proposed modification of nodal status in AJCC esophageal cancer staging system. Ann Thorac Surg 84:365-375

27. Yuan S, Yu Y, Chao KSC, et al. (2006) Additional value of PET/ $\mathrm{CT}$ over PET in assessment of locoregional lymph nodes in thoracic esophageal squamous cell cancer. Nucl Med 47:1255-1259

28. Hs I, Kim SJ, Kim IJ, Kim K (2012) Predictive value of metabolic tumor volume measured by $18 \mathrm{~F}-\mathrm{FDG}$ PET for regional lymph node status in patients with esophageal cancer. Clin Nucl Med 37:442-446

29. Nieman DR, Peyre CG, Watson TJ, et al. (2015) Neoadjuvant treatment response in negative nodes is an important prognosticator after esophagectomy. Ann Thorac Surg 99:277-283

30. Manabe O, Hattori N, Hirata K, et al. (2013) Diagnostic accuracy of lymph node metastasis depends on metabolic activity of the primary lesion in thoracic squamous esophageal cancer. J Nucl Med 54:670-676

31. Foley KG, Lewis WG, Fielding P, et al. (2014) N-staging of oesophageal and junctional carcinoma: is there still a role for EUS in patients staged N0 at PET/CT? Clin Radiol 69:959-964

32. Devadas M, Mittal A, Lin M, et al. (2015) FDG-PET nodal staging does not correlate with histopathological nodal stage for oesophageal cancers. Int J Surg 20:113-117 